\title{
The presheaf of conditional
}

expectations, a bridge between probability and intuitionistic

\section{truth value}

Jean-Pascal Laedermann, PhD1

Independent researcher

November 2021

English translation Michelle Bailat-Jones²

Boolean characteristic functions

Heyting characteristic cribles

\footnotetext{
1 pec@laedus.org

2 www.mbjtranslation.com
} 
Abstract 3

Introduction 4

Concepts $\quad 5$

Contexts 6

As Boolean algebras $\quad 6$

As vector spaces 6

Classic and intuitionistic validities 9

Interior, border, and exterior of a proposition 11

Carrying capacity 11

Reservation 12

Minimal contexts and construction of cribles 13

Description of the process 14

Quantile-contexts 15

Illustrations 16

Case $\mathrm{D}=2 \quad 16$

Case $\mathrm{D}=3 \quad 17$

Conclusion $\quad 22$

Selected bibliography 23 


\section{Keywords}

category, topos, presheaf, probability, validity, truth, conditional expectation, measurement, quantum mechanics, information, entropy, reduction, collapse, projection, logic, algebra, Wiener, Bayes, Boole, Heyting, Brownian motion, filter, crible, capacity, reservation, context

\section{Abstract}

Research for a theory of quantum gravity has recently led to the use of presheaf topos. Quantum uncertainty is linked to the truth values of intuitionistic logic. This paper proposes transposing this model into a classic probability context, that of conditional mathematical expectations. A simulation of Brownian motion is offered for illustrative purposes. 


\section{Introduction}

The notion of uncertainty can be modeled in at least three ways. The first and standard model works according to Kolmogorov probability axioms. In this approach, an objective definition of probability is based on the repetition of independent experiences in which frequencies converge. It is founded upon Boolean logic. Quantum mechanics is a second approach, in which repetition becomes problematic because of the influence of the measurement on the observed system. In this approach, probability is obtained in a projectorial manner. Finally, a third approach is the one called topos, in which uncertainty takes the form of truth values within a Brouwer-Heyting logic.

The state of a system on which an observer has only partial information is modelled in a classical way using a probability space. In information theory, a question, or a measurement, is translated by a finite partition of this space, whose elements represent propositions corresponding to different outcomes, or observable responses. This means that the Boolean algebra generated by this partition is comprised of the full set of propositions the observer can articulate about the system. This algebra is the common context of observation, and the system's response becomes one of the algebraic elements instantiated by the measurement. This instantiation then makes it possible to estimate probability distributions for approximations of the observables connected to the system. This paper will propose associating them with the truth values of a given Heyting algebra.

An application to Wiener processes will be developed.

This work is directly inspired by the different developments which appeared in the framework of quantum mechanics oriented toward topos. Here, the conceptualization achieved by researchers such as Johan Baez, Chris J. Isham, Andreas Döring [1], J. Gutt [1.1] and Cecilia Flori [2, 2.1] is reworked from a classical point of view. The conclusion of the paper will develop this parallel once the necessary mathematical objects have been defined. 


\section{Concepts}

\section{Conditional mathematical expectation}

Let $(\Theta, \mathscr{A}, P)$ be a probability space. The random variables which can be absolutely integrated on this space will be called observables.

$\downarrow \mathscr{A}$ designates all of its finite subalgebras. This type of subalgebra is a graining of $\Theta$, which gets finer the closer to $\mathscr{A} . \downarrow \mathscr{A}$ is a bounded lattice. The infimum $(\wedge)$ of two algebras will be their intersection, and the supremum $(\mathrm{V})$ the smallest $\sigma$-algebra that contains them. The order relationship, which here is the inclusion, will be noted $\hookrightarrow$. The bounds of this lattice are $\alpha_{\varnothing}=\{\varnothing, \Theta\}$ and $\mathscr{A}$, the coarsest algebra and the limit algebra. Let $\alpha \in \downarrow \mathscr{A}$ and $X: \Theta \rightarrow \mathbb{R}$ be an observable. The conditional mathematical expectation $\mathbb{E}(X \mid \alpha)[3,3.1]$ is an $\alpha$-mesurable observable that verifies

$$
\int U X d P=\int U \mathbb{E}(X \mid \alpha) d P
$$

for any $\alpha$-mesurable observable $U . \mathbb{E}(X \mid \alpha)$ is an approximation of $X$ on $\alpha$. If $\alpha$ is finite, this approximation is a step function, constant on the atoms of $\alpha$. An important property of this expectation is that if $\beta \hookrightarrow \alpha$, then

$$
\mathbb{E}(\mathbb{E}(X \mid \alpha) \mid \beta)=\mathbb{E}(X \mid \beta)
$$

\section{Presheaf of conditional expectations}

The set of the subalgebras $\downarrow \mathscr{A}$ fitted with its order relationship $\hookrightarrow$ canonically becomes a poset category whose objects are elements and arrows the order relationship. This makes it possible to define $\widehat{\downarrow \mathscr{A}}=\mathbf{S e t}^{\left\lfloor\mathscr{A}^{o p}\right.}$ which is a presheaf topos.

The property (i) leads to understanding the conditional expectation $\mathbb{E}$ as an object of this topos. By definition, $\mathbb{E} \alpha$ would be the set of the $\alpha$-mesurables observables, and its action on the arrows defined by $X \in \mathbb{E} \alpha, \mathbb{E}(\alpha \hookleftarrow \beta) X=\mathbb{E}(X \mid \beta)$. By extension $\mathbb{E} \mathscr{A}$ would be the entire set of $P$-integrable random variables. It is easily seen using (i) that this gives a contravariant functor. This functor will be called the presheaf of conditional expectations associated to the probability space $(\Theta, \mathscr{A}, P)$. 


\section{Contexts}

\section{As Boolean algebras}

The finite subalgebras $\alpha$ of $\mathscr{A}$ are generated by measurable partitions $\Theta=\sum_{\Delta \in \pi \alpha} \Delta$ and will be named contexts of observation. The elements of $A \in \alpha$ will be associated to the propositions of this context, and especially those of the partition, its atoms, will be the elementary propositions of the context.

Information theory [4] defines a question by a finite partition of the set of the states $\theta \in \Theta$. Each element of this partition is an outcome associated to a value of the response. An outcome can also be seen as a proposition, able to take the value $T$ ou $\perp$.

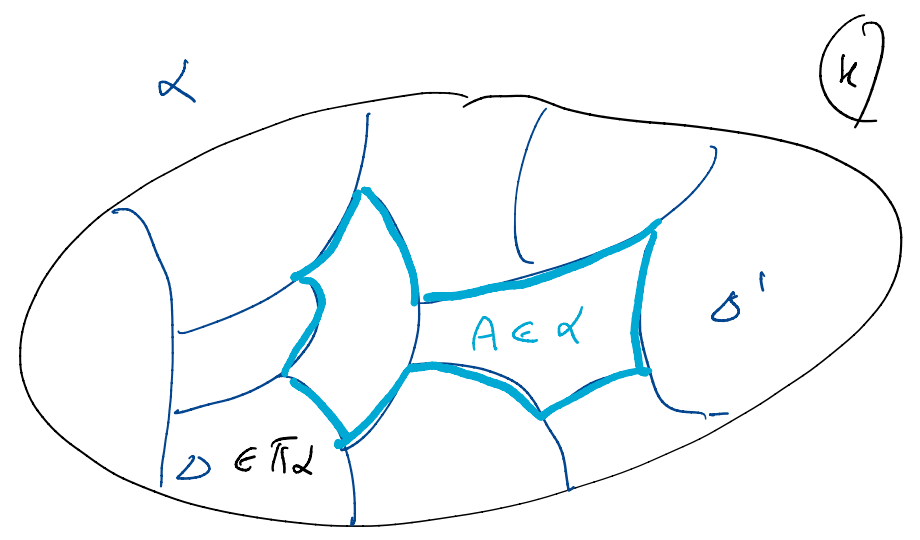

\section{As vector spaces}

In the finite case, the presheaf $\mathbb{E}$ takes a particularly simple form. Indeed, the sets of observables $\mathbb{E} \alpha$ are naturally equipped with a vector space structure on $\mathbb{R}$, even with a commutative algebra structure.

The canonical basis vectors of $\mathbb{E} \alpha$ are the characteristic functions of the partition elements $\left(\chi_{\Delta}\right)_{\Delta \in \pi \alpha}$ and the observables become vectors whose dimension is the cardinal of the partition.

The conditional probabilities $P \mid \Delta$ make it possible to construct the dual canonical basis $\left(\chi^{\Delta}\right)_{\Delta \in \pi \alpha}$, a set of linear forms acting on an observable $X$ according to $\chi^{\Delta} X=E(X \mid \Delta)=\frac{1}{P(\Delta)} \int_{\Delta} X d P$.

This results in the decomposition of the identity $I d_{\mathbb{E} \alpha}=\sum_{\Delta \in \pi \alpha} \chi_{\Delta} \chi^{\Delta}$. 
If $\beta \hookrightarrow \alpha$, the vector subspaces are included: $\mathbb{E} \beta \hookrightarrow \mathbb{E} \alpha$.

It is easily confirmed that $\mathbb{E}(\alpha \hookleftarrow \beta)$ is the projector $\sum_{\Gamma \in \pi \beta} \chi_{\Gamma} \chi^{\Gamma}: \mathbb{E} \alpha \rightarrow \mathbb{E} \beta$.

In the context $\alpha$, the integral built on the restriction of probability (i.e. the simple mathematical expectation) becomes the linear form $E=\sum_{\Delta \in \pi \alpha} P(\Delta) \chi^{\Delta}$.

The $\mathbb{E} \alpha$ subalgebras thus give a vectorial stratification of the basic space $\mathbb{E} \mathscr{A}$. It is worth noting that the projector $\sum_{\Delta \in \pi \alpha} \chi_{\Delta} \chi^{\Delta}$ is well defined across all the observables of $\mathbb{E} \mathscr{A}$, it is only in its restriction to $\mathbb{E} \alpha$ that it becomes a decomposition of identity.

It is well known that $\mathbb{E} \mathscr{A}$ can be equipped with a Hilbert space structure (real here) with the scalar product $\langle X \mid Y\rangle=\int X Y d P$. The basis $\left(\chi_{\Delta}\right)_{\Delta \in \pi \alpha}$ is orthogonal for this product. It is possible to normalize it by defining, in the style of Dirac, the kets $|\Delta\rangle=\frac{1}{\sqrt{P(\Delta)}} \chi_{\Delta}$ and the bras $\langle\Delta|=\sqrt{P(\Delta)} \chi^{\Delta}$.

It's possible to further push the analogy to quantum mechanics. The effect of measuring an observable $X$ in a context $\alpha$ projects it onto its conditional expectation $\mathbb{E}(X \mid \alpha)$. This new observable is discrete and takes the value $X^{\Delta}$ with the probability $P(\Delta)$. 


\section{Subobjects and truth values}

Let $D$ be an arbitrary set of observables. The application $D \mapsto s_{D}$ given by $s_{D} \alpha=\{\mathbb{E}(X \mid \alpha) \mid X \in D\}$ is a subobject of $\mathbb{E}$. Indeed, $(\forall \alpha)\left(s_{D} \alpha \subseteq \mathbb{E} \alpha\right)$ and for $X \in D,\left(\alpha \hookleftarrow \beta, E(X \mid \alpha) \in s_{D} \alpha\right) \Longrightarrow \mathbb{E}(X \mid \beta) \in s_{D} \beta$.

If $X, Y$ are two observables, the equivalence relation $X \stackrel{\alpha}{\sim} Y$ is defined by $\mathbb{E}(X \mid \alpha)=\mathbb{E}(Y \mid \alpha)$. It expresses the fact that $X$ and $Y$ are equal in context $\alpha$ (otherwise stated at the approximation level $\alpha$ ). Note that $X \stackrel{\alpha}{\sim} Y \Longrightarrow E X=E Y$.

The subobject classifier of the topos $\widehat{\downarrow \mathscr{A}}$ is given by the functor $\Omega$ where $\Omega \alpha$ is the set of cribles over $\alpha$. For an observable $X \in \mathbb{E} \alpha$ and a $s_{D}$ type subobject, the truth value of the $« X \in s_{D}$ in context $\alpha$ » relationship can be written

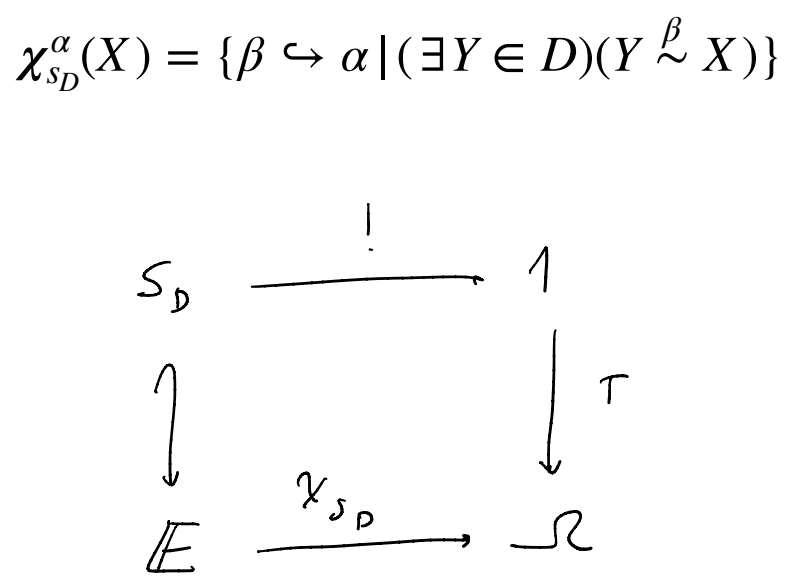

These truth values are elements of a Heyting algebra. In order to differentiate between the different types of characteristics, the cribles will be noted in bold typeface. 


\section{Measurement}

Let's suppose that an observer is in the presence of a system composed of independent identical $M$ subsystems whose distribution over the states is $P$. To be clear, let's name these subsystems particles. One of these particles is pointed. This system is canonically modelled by the space $\left(\Theta^{M}, \mathscr{A}^{M}, P^{\otimes M}\right)$, and a state will be a vector $\boldsymbol{\theta}=\left(\theta_{i}\right)_{i \in M}$. Let's also define $\tilde{\boldsymbol{\theta}}=\left\{\theta_{i} \mid i \in M\right\}$. A measurement of this system, done in a context $\alpha$ will give a result $G_{\boldsymbol{\theta}}^{\alpha}=\Sigma\left\{\Delta \in \pi \alpha \mid(\exists i \in M)\left(\Delta \ni \theta_{i}\right)\right\}^{3}$. This response is a proposition of $\alpha$ which can also be written as $\bigcap\{A \in \alpha \mid A \supseteq \tilde{\boldsymbol{\theta}}\}$. The observer knows neither the number of particles in each outcome, nor the outcome in which the pointed particle is located.

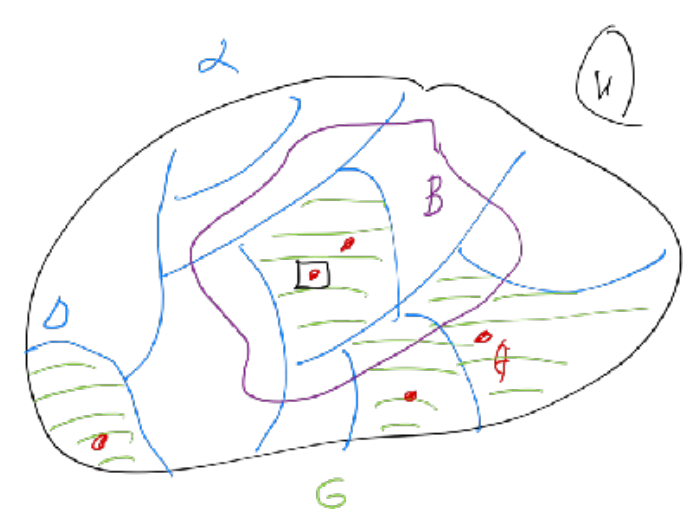

\section{Classic and intuitionistic validities}

The observer, having fixed its context $\alpha$, could be interested in the effect of the result $G_{\boldsymbol{\theta}}^{\alpha}$ of a measurement on a given $B \in \mathscr{A}$ proposition. For example, the observer could wonder how much confidence to attribute to the proposition « The pointed particle is in $B$, given that the response of the system is $G_{\boldsymbol{\theta}}^{\alpha}$ ".

The classical solution is given by the conditional probability $P\left(B \mid G_{\boldsymbol{\theta}}^{\alpha}\right)$.

Another possibility is the following.

Let $\mathscr{V}(G)=\{U \in \mathscr{A} \mid U \supseteq G\}$. This is a filter that gives propositions validated by $G$. If these propositions are represented by their characteristic functions, the subobject $s_{\mathscr{V}\left(G_{\theta}^{\alpha}\right)}$ of $\mathbb{E}$ can be constructed. To this subobject is associated its characteristic arrow $\chi_{S_{\mathscr{V}\left(G_{\theta}^{\alpha}\right)}}$ which makes it possible to obtain the crible $\chi_{S_{\mathscr{V}\left(G_{\theta}^{\alpha}\right)}^{\alpha}}^{\alpha}(B)$. This is an element of the classifier $\Omega \alpha$, therefore a truth value of intuitionistic logic.

3 The union is disjointed because it bears upon the elements of a partition. 
Uncertainty results from a thickening of the state $(\alpha, \boldsymbol{\theta}) \mapsto G_{\boldsymbol{\theta}}^{\alpha}$. It is evaluated in a probabilistic manner by the conditioning $P \mid G_{\theta}^{\alpha}$, and in an intuitionistic manner by the instantiation of $\alpha$ of the subject associated to the validation filter $s_{\mathscr{V}\left(G_{\theta}^{\alpha}\right)}$. Nevertheless, note that the application $\alpha \mapsto s_{\mathscr{V}\left(G_{\theta}^{\alpha}\right)}^{\alpha}$ does not define a subobject of $\mathbb{E}$.

\section{Definitions}

For a given $\boldsymbol{\theta}$ and $P$, the intuitionistic validity in the context $\alpha$ of a proposition $B \in \mathscr{A}$ is given by the crible

$$
v_{I}^{\alpha} B=\left\{\beta \hookrightarrow \alpha \mid(\exists U \in \mathscr{A})\left(U \supseteq G_{\boldsymbol{\theta}}^{\alpha}, U \stackrel{\beta}{\sim} B\right)\right\}
$$

and the classical validity by the number

$$
v_{C}^{\alpha} B=P\left(B \mid G_{\theta}^{\alpha}\right)
$$

\section{Interpretation of intuitionistic validity}

For an observer equipped with a context $\alpha$, two equivalent propositions $U \stackrel{\alpha}{\sim} B$ are indistinguishable. The crible $v_{I}^{\alpha} B$ thus contains all the subcontexts for which $B$ is validated up to equivalence.

The equivalence present in the crible can be written

$$
U \stackrel{\beta}{\sim} B \Longleftrightarrow(\forall \Gamma \in \pi \beta)(P(U \mid \Gamma)=P(B \mid \Gamma))
$$

Especially $v_{I}^{\alpha} B=T$ leads to $(\forall \Delta \in \pi \alpha)(P(B \mid \Delta)=P(U \mid \Delta))$, therefore $(\forall \Delta \in \pi \alpha)(P(B \cap \Delta)=P(U \cap \Delta))$. Since $G_{\boldsymbol{\theta}}^{\alpha} \in \alpha$, we get $P\left(B \cap G_{\boldsymbol{\theta}}^{\alpha}\right)=P\left(G_{\boldsymbol{\theta}}^{\alpha}\right)$, which gives $B \stackrel{\text { p.s. }}{\supseteq} G_{\boldsymbol{\theta}}^{\alpha}$.

The intuitionistic character of the logic is easily seen in the following situation, where $v_{I}^{\alpha}\left(B \cup B^{\prime}\right)=\top$, without having $v_{I}^{\alpha} B=\top$ nor $v_{I}^{\alpha} B^{\prime}=\top$.

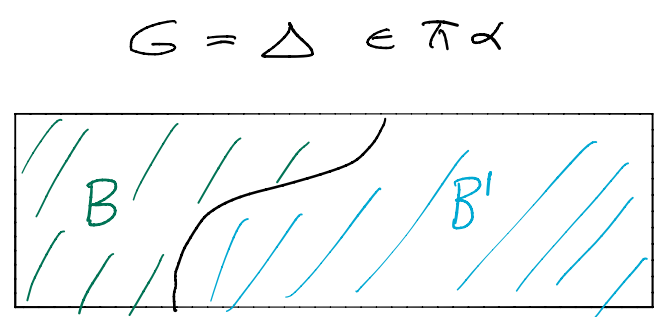




\section{Calculating cribles}

Everything is about finding the propositions $U$ approximating $B$ which are validated by the measurement.

Let's introduce a few practical tools.

Interior, border, and exterior of a proposition

Let $B \in \mathscr{A}$ be an arbitrary proposition. Seen in $\alpha$, it brings about three propositions belonging to the context :

- its interior $\stackrel{\circ}{\alpha} B=\Sigma\{\Delta \in \pi \alpha \mid \Delta \subseteq B\}$

- its exterior $\stackrel{e}{\alpha} B=\Sigma\{\Delta \in \pi \alpha \mid \Delta \subseteq \complement B\}$

- its border $\partial \alpha B=\Sigma\{\Delta \in \pi \alpha \mid(\Delta \cap B \neq \varnothing) \wedge(\Delta \cap C B \neq \varnothing)\}$

\section{Notes}

- $\partial \alpha B=\varnothing \Longleftrightarrow B \in \alpha$

- $\partial \alpha B=\partial \alpha \complement B$

$\cdot \beta \hookrightarrow \alpha \Longrightarrow \partial \alpha \circ \partial \beta=\varnothing$

- $\partial(A \cap B)=\partial A \cap \partial B+\partial A \cap B+A \cap \partial B$

- $\partial(A \cup B)=\partial A \cup \partial B-\partial A \cup B-A \cup \partial B$

\section{Carrying capacity}

\section{Definition}

The carrying capacity related to a context $\alpha$ of a part $R$ of the border $\partial \alpha B$ is defined by

$$
\kappa(R)=P(R \cap B)
$$

This part is considered compatible with the measurement $G$ iff

$$
(\exists U)(G \cap R \subseteq U \subseteq R, \kappa(R)=P(U))
$$

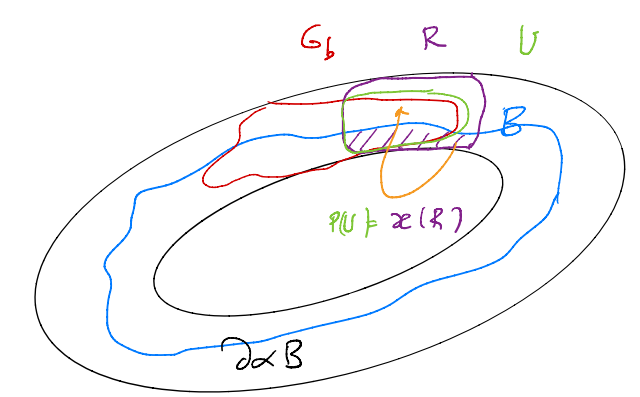




\section{Note}

$U$ does not necessarily belong to $\alpha$.

The idea is to be able to replace the part of $B$ on the border by a subset of sufficient probability to accommodate the outcomes of the measurement located there (cf. below).

\section{Reservation}

\section{Definition}

An ordered pair $(G, \bar{G})$ will be a reservation iff

$$
(G, \bar{G}) \in \alpha^{2}, G \in \stackrel{e}{\alpha} B, \bar{G} \in \stackrel{\circ}{\alpha} B, P(G)=P(\bar{G})
$$

\section{Note}

The idea is that the interior of $B$, in addition to the interior part of the measurement, must be able to accommodate its exterior part (cf. below). 


\section{Minimal contexts and construction of cribles}

The result of the measurement $G_{\boldsymbol{\theta}}^{\alpha}$, which will be noted as $G$ to simplify the notation, is partitioned into three parts: $G_{e}=G \cap \stackrel{e}{\alpha} B, G_{b}=G \cap \partial \alpha B$ et $G_{i}=G \cap \stackrel{\circ}{\alpha} B$.

To get $v_{I}^{\alpha} B \neq \perp$, note that the border of $B$ must be compatible with $G_{b}$. Let $U_{b}$ be the element guaranteeing this compatibility, if it exists.

\section{Theorem}

- The minimum elements of the crible $v_{I}^{\alpha} B$ are characterized by the compatibility of the border and the existence of a reservation $\left(G_{e}, \bar{G}_{e}\right)$ such as $\bar{G}_{e} \cap G_{i}=\varnothing$

- An approximation $U \stackrel{\beta}{\sim} B$ is given by $U=U_{b}+G_{e}+\stackrel{\circ}{\alpha} B-G_{i}$

- The atoms of $\beta$ are given by $\pi \beta=\left\{\partial \alpha B, G_{e}+\bar{G}_{e}, \complement\left(\partial \alpha B+G_{e}+\bar{G}_{e}\right)\right\}$

- The other elements of the crible are obtained by fractioning the border into compatible parts, fractioning the reservation into subreservations, and a fractioning of the complement into elements of $\alpha$

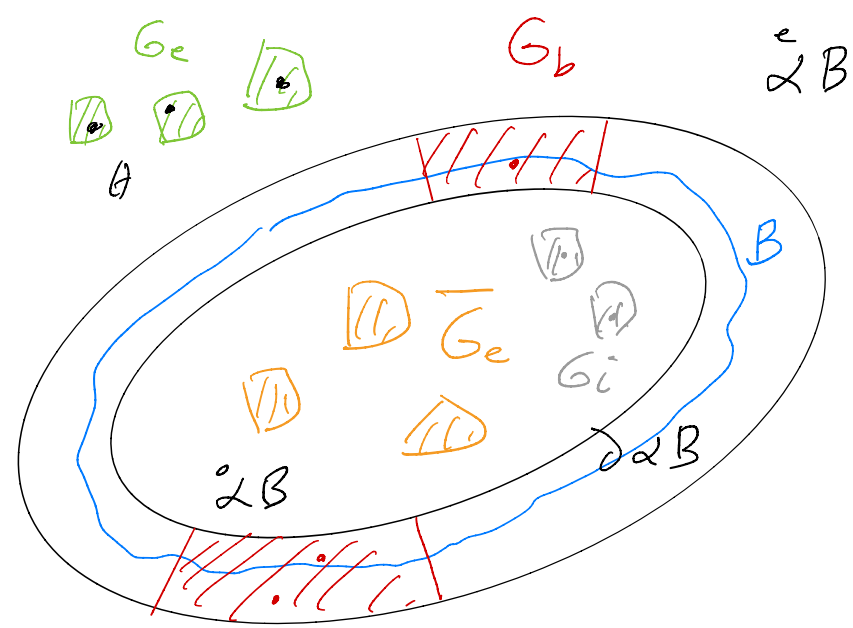

The illustration above shows that $\partial \alpha B$ et $G_{e}+\bar{G}_{e}$ appear as orthogonals.

\section{Corollaries}

If the measurement can be found entirely on the interior of the proposition, its classical validity equals 1 and its intuitionistic validity is $T$. However, if the measurement can be found on the exterior of the proposition, the classic validity is 0 , while the intuitionistic validity can likely be different from $\perp$. For this to work the reservation must be possible. There is a connection between this phenomenon and the quantum tunnel effect, for example, in which a particle with insufficient energy manages to cross a potential barrier.

The reservation $R=G_{e}+\bar{G}_{e}$ gives $\partial \beta B=\partial \alpha B+R$. This can be seen as an intrication. 


\section{Application: considering Brownian motion}

\section{Description of the process}

Here we propose an illustration of certain preceding concepts using a consideration of Brownian motion. Independent $M$ particles move about in $\mathbb{R}^{D}$ according to Wiener's stochastic process whose transition probabilities are

$$
p_{\Delta t}\left(\theta, d \theta^{\prime}\right)=\bigwedge_{i \in D} \frac{1}{\sqrt{2 \pi \Delta t}} e^{\frac{\left(\theta^{i}-\theta^{i}\right)^{2}}{2 \Delta t}} d \theta^{i}
$$

At each instant $t$, the system is described by a state $\boldsymbol{\theta} \in \mathbb{R}^{D^{M}}$. The state is measured in a context $\alpha$ given by a finite Borel-measurable partition $\mathbb{R}^{D}$. The result of the measurement is simply the union of outcomes in which the particles are found, meaning $G_{\boldsymbol{\theta}}^{\alpha}=\Sigma\left\{\Delta \in \pi \alpha \mid(\exists i \in M)\left(\theta_{i} \in \Delta\right)\right\}$. The observer thus only approximately measures the positions of the particles and does not know their number in each of the context's outcomes. The observer's a priori knowledge of the systems at a time $t$ is a probability distribution $P_{t}$ over $\mathbb{R}^{D}$. The evolution of the system is initialized with the particles placed at the origin, i.e. $P_{0}=\delta_{0}$.

The evolution then continues in stages. For the stage beginning at time $t$, the particles are localized in $\boldsymbol{\theta}_{t}$, and the observer has information that is represented by the distribution $P_{t}$. The stage lasts for a duration $s$, at the end of which each particle has evolved randomly to end up in a position sampled by $P_{t+s}^{i}(d \theta)=\int p_{s}\left(\theta_{t}^{i}, d \theta\right), i \in M$, which gives the state $\boldsymbol{\theta}_{t+s}$. For the observer, $P_{t}$ has become $P_{t+s}\left(d \theta^{\prime}\right)=\int p_{s}\left(\theta, d \theta^{\prime}\right) P_{t}(d \theta)$. This probability makes it possible to calculate $v_{I}$ and $v_{C}$, while keeping track of the outcome $G_{\boldsymbol{\theta}_{t+s}}^{\alpha}$ of the context. This response enables the observer to refine its information, which moves from $P_{t+s}$ before the measurement to $P_{t+s} \mid G_{\boldsymbol{\theta}}^{\alpha}$ after the measurement. The support of the latter being smaller, we can speak of collapse. This distribution thus serves as prior to the observer for the following stage.

This references quantum measurement, in which the wave function is reduced through projection over the subspace associated to the result. Nevertheless, in this case the measurement does not influence the state of the system. 


\section{Quantile-contexts}

In order to enable a simulation of the process, we will use specific contexts which can be considered generalized quantiles. Each pair $(\alpha, P)$ is associated with an entropy $H(\alpha)=-\sum_{\Delta \in \pi \alpha} P(\Delta) \ln P(\Delta)$ that gives the average information that can be obtained with the context $\alpha$. It is well known that this entropy is maximum when all its outcomes have the same probability. This is possible here because the $P_{t}$ are of a continuous density with respect to Lebesgue's measure. The following is a simple way of proceeding. Select a basis $\left(e_{i}\right)_{i \in D}$ of $\mathbb{R}^{D}$, not necessarily orthogonal, and for each vector $e_{i}$ of this basis, a number of divisions $L_{i}$. Beginning with $e_{0}$, look for the hyperplanes parallel to the index vectors above 0 which cut $P_{t}$ into $L_{0}$ divisions of equal probability. Then in each of these divisions, make the same type of division in the direction $e_{1}$, and so on and so forth. The final result is a division of $\mathbb{R}^{D}$ into $N_{I}=\prod_{i \in D} L_{i}$ issues, outcomes, which are hyperparallelograms of probability $\frac{1}{N_{I}}$. Stopping at $D=1$ gives the usual definition of quantiles. These particular contexts will be named quantile-contexts. Note that this division depends on the order of the vectors.

The intuitionistic validity can be easily obtained in the case of quantile-contexts. All the outcomes have the same probability $u=\frac{1}{N_{I}}$. So that for $v_{I}^{\alpha} B$ to be non-empty, it just has to have $P(\stackrel{\circ}{\alpha} B) \geqslant P\left(G_{e}\right)+P\left(G_{i}\right)$ and $\kappa(\partial \alpha B) \geqslant P\left(G_{b}\right)$. 


\section{Illustrations}

Case $\mathrm{D}=2$

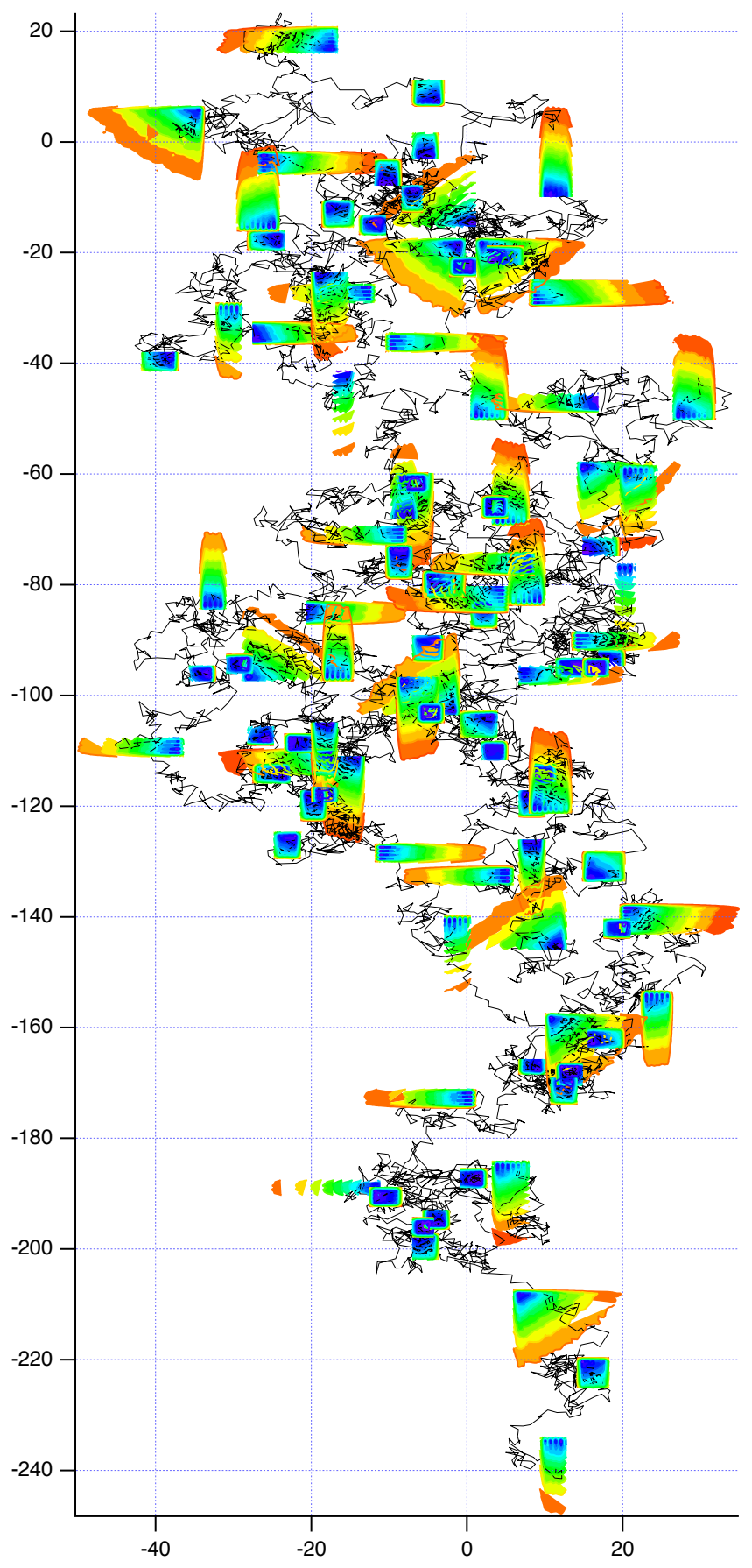

Brownian motion of 100 observations distant from 100 steps with a quantile-context of 49 outcomes. The leveling curves give the posterior divisions (collapses) produced by each measurement. 


\section{Case $\mathrm{D}=3$}

\section{TRAJECTORIES AND DETECTIONS}

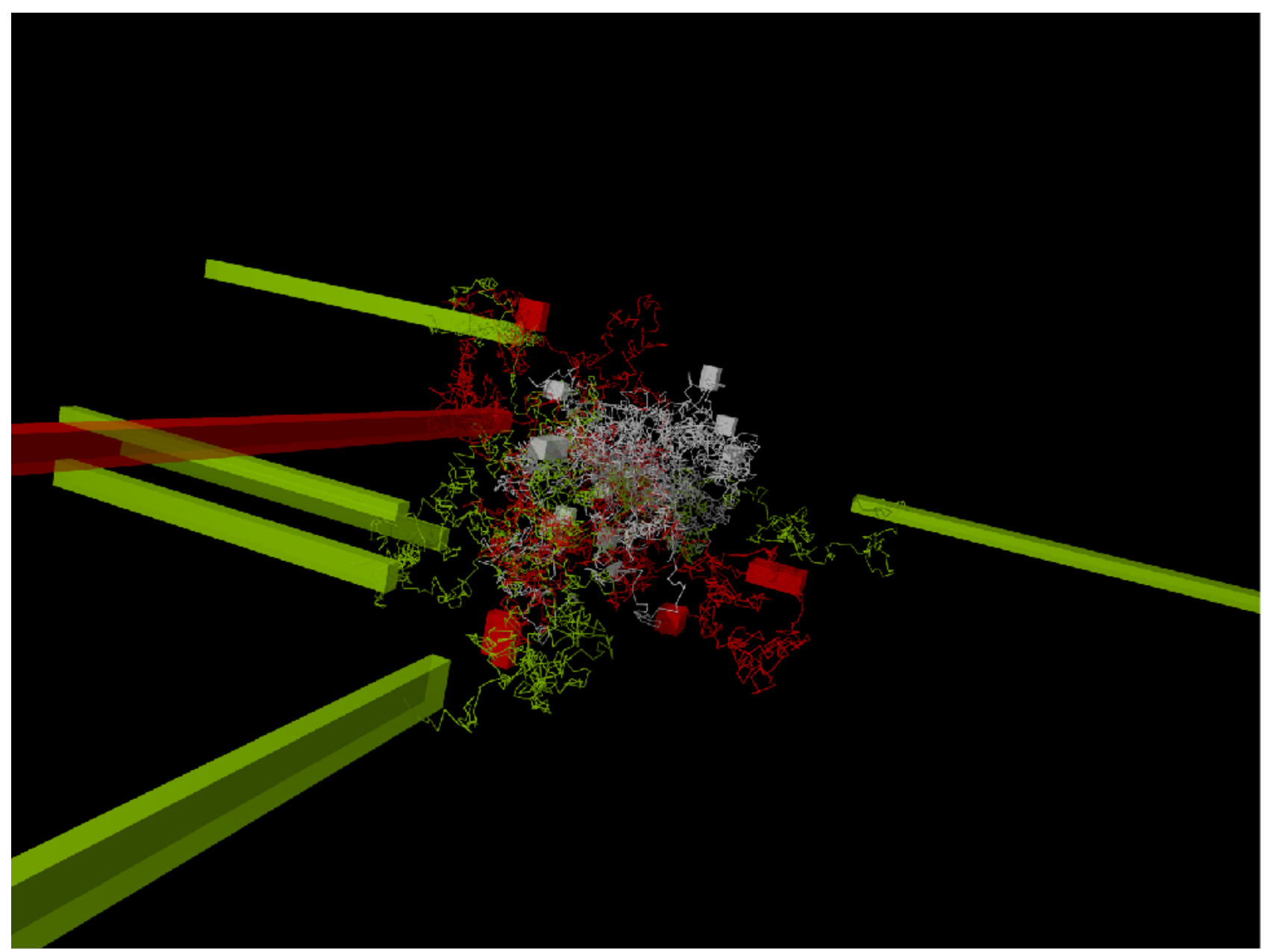

Detection of particles by quantile-context. The green outcomes are in the exterior of a cube, the red are in its border and the white in its interior. The parallelograms of the extremities of the context have been truncated, because they are unbounded. Nevertheless, their probability is equal to the others 


\section{BORDERS}

We looked at the borders of the propositions $B$ of type « to be in a measurable region of $\mathbb{R}^{D}$ ». The following figures were obtained for the interior of a cube, a torus, a pair of ellipsoids, and the upper part of the surface $\theta^{2}>\sin \theta^{0}$. Animations of these images are available at https://laedus.org

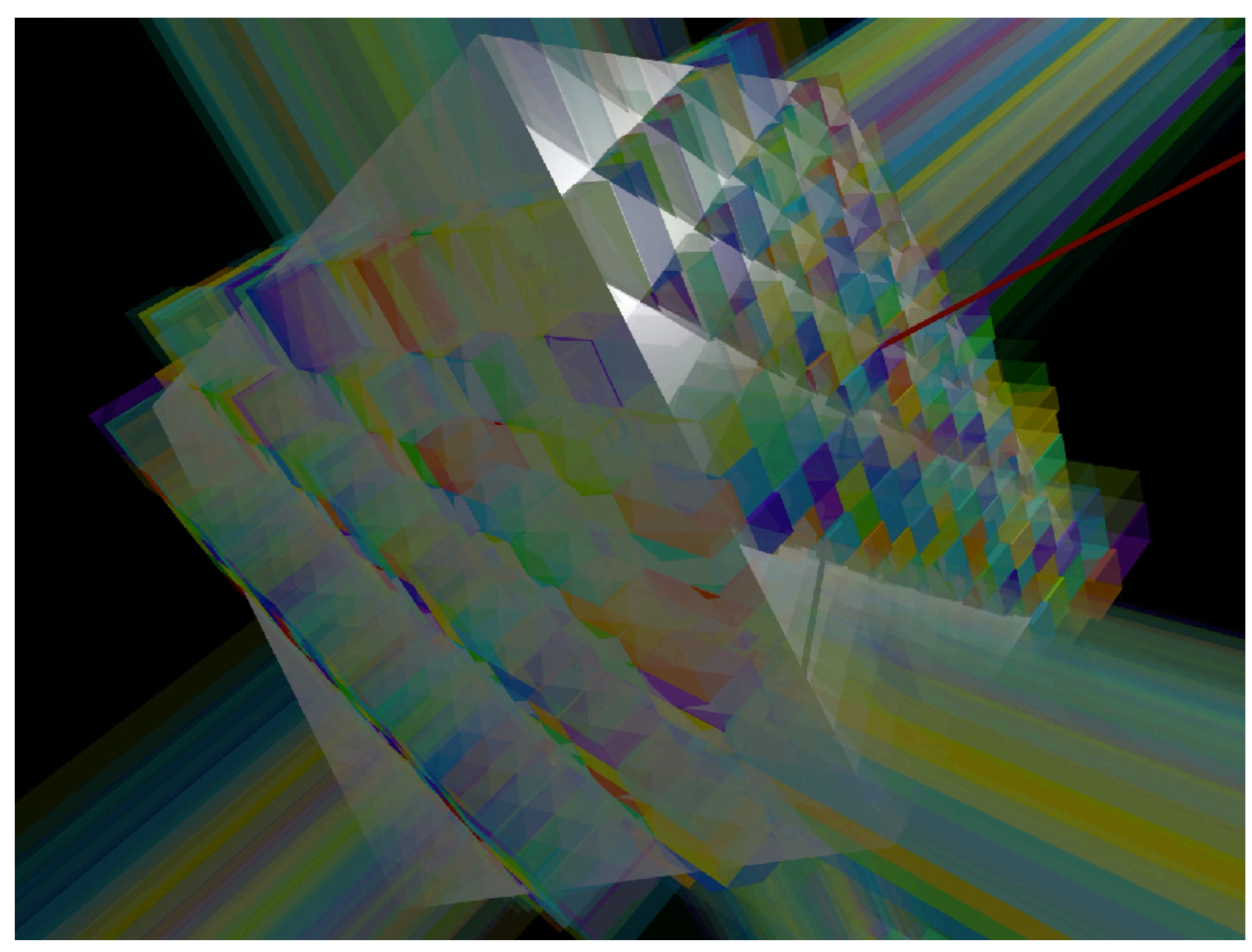

Border of a cubic proposition 


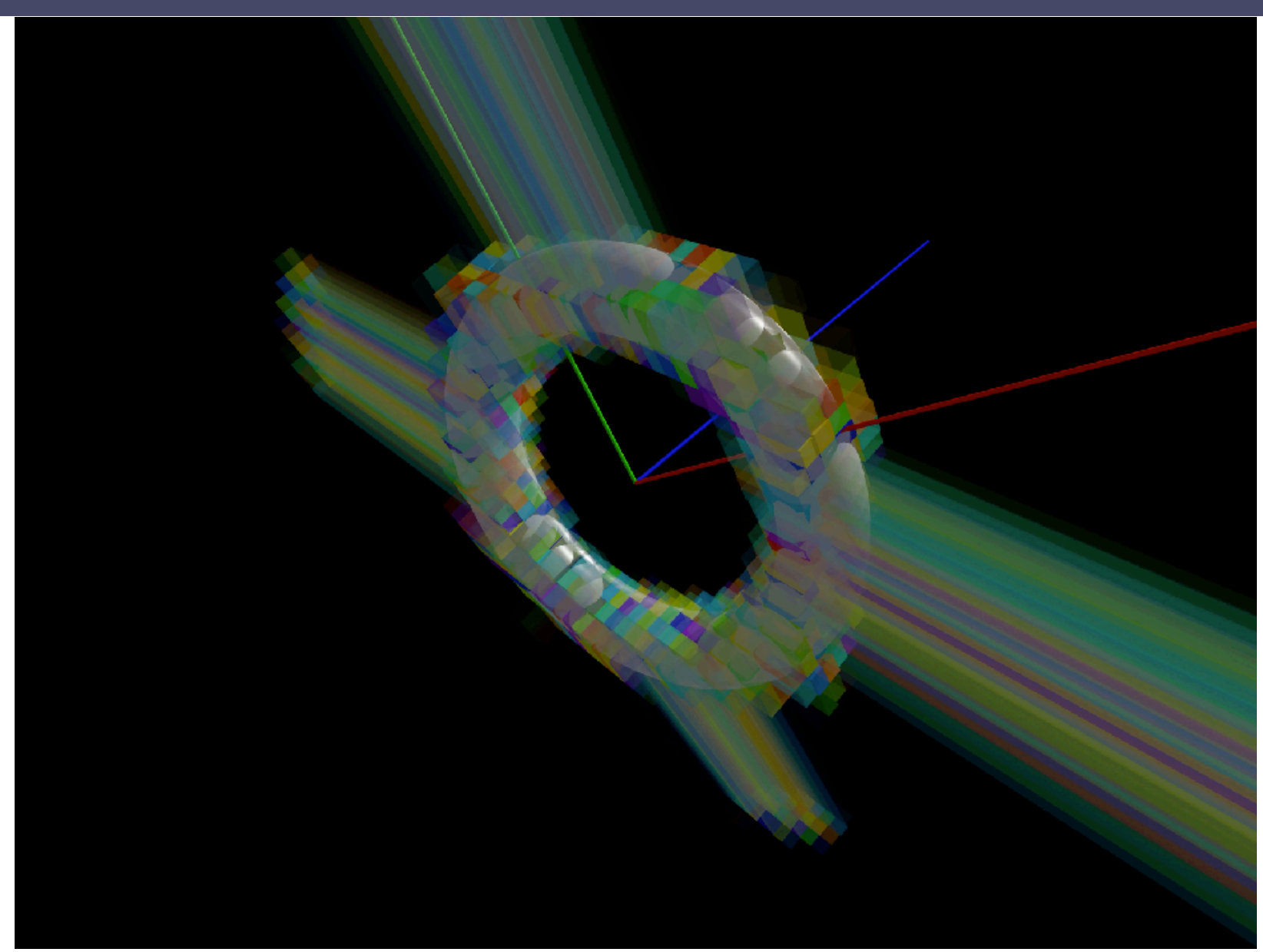

Borders of toric and double-elliptic propositions

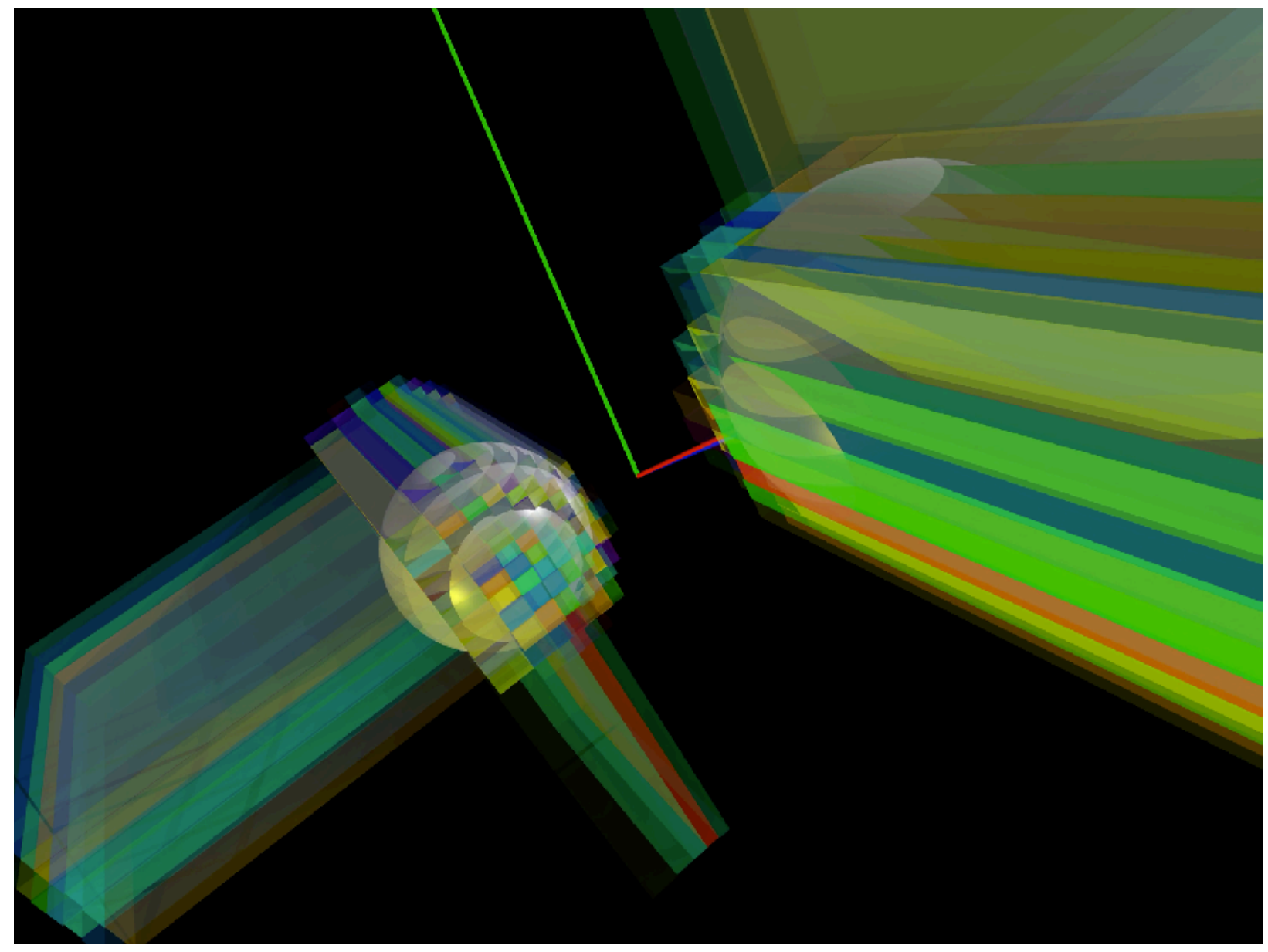




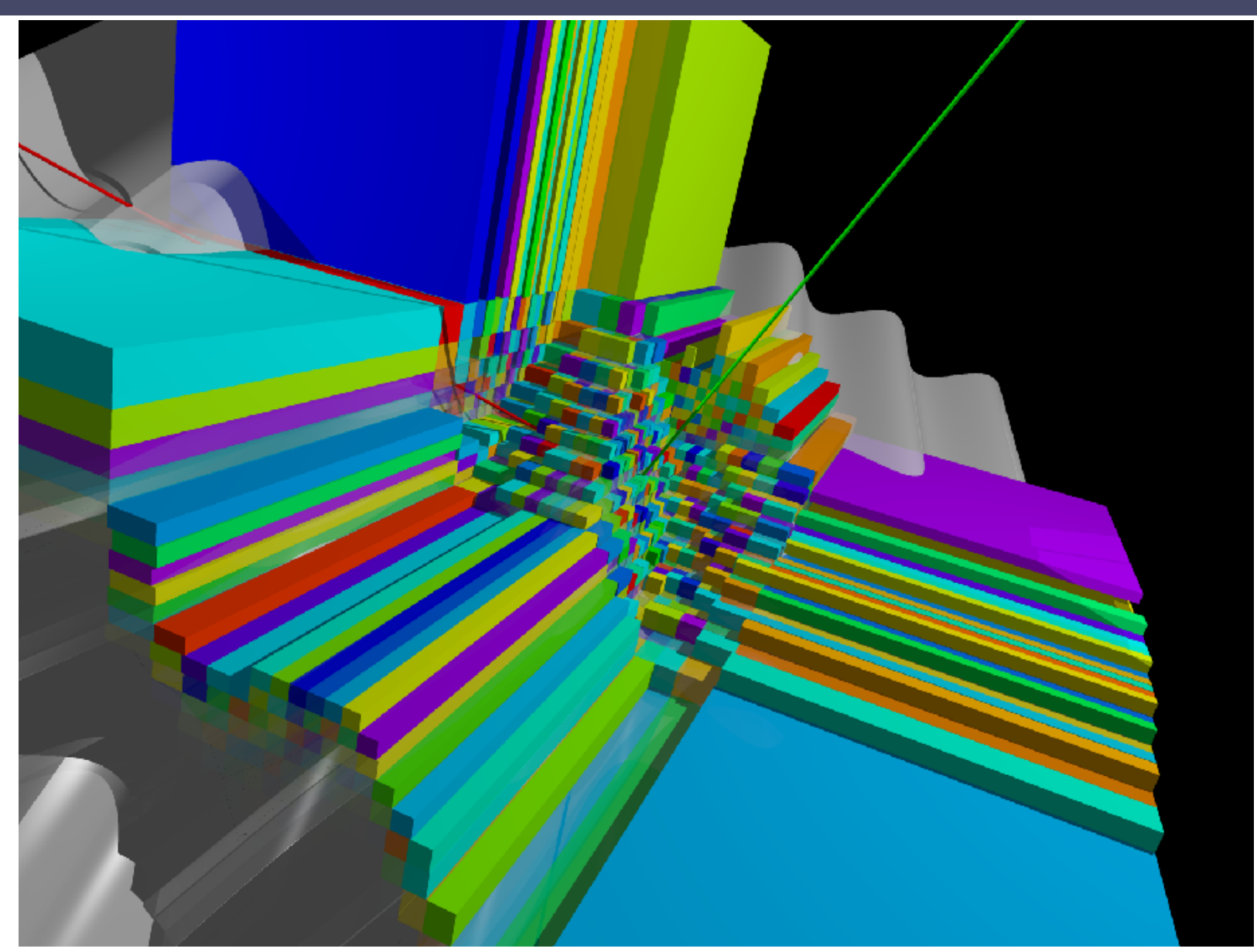

Border of a sinusoïdal proposition

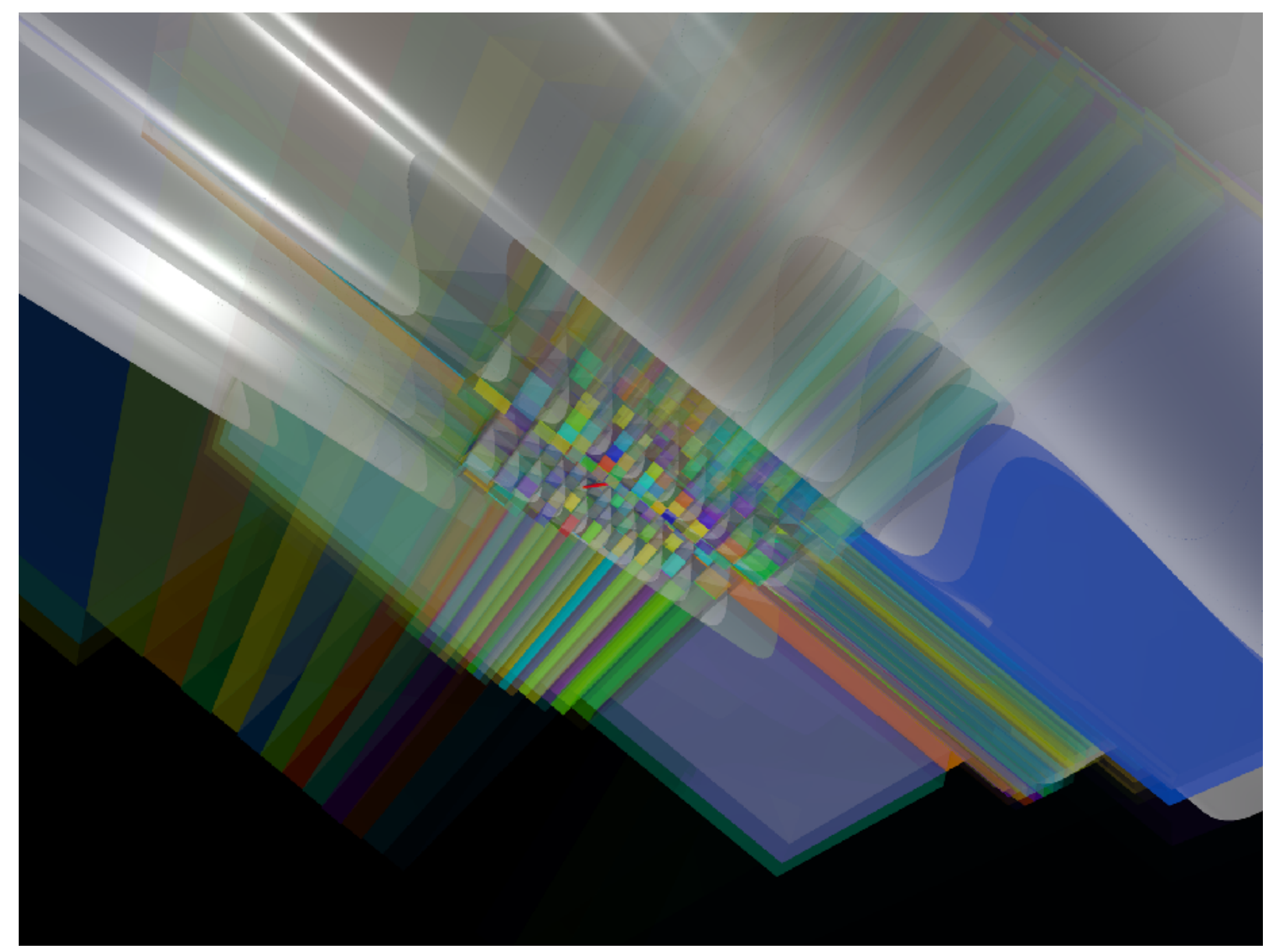




\section{Parallel with toposic modeling of quantum mechanics}

The mathematical objects associated with the presheaf of conditional expectations (PCE) are directly inspired by those coming from Isham and Döring's (ID) quantum model.

\begin{tabular}{|c|c|}
\hline PCE & ID \\
\hline$\downarrow \mathscr{A}$ & $\mathscr{V}(\mathscr{H})$ \\
\hline$\alpha$ & $V$ \\
\hline$\chi_{B}, B \in \mathscr{A}$ & $\mathscr{J}(\mathscr{H})$ \\
\hline$P \in \mathfrak{M}_{1} \mathscr{A}$ & $\psi \in \dot{\mathscr{H}}$ \\
\hline $\mathbb{E}$ & $\mathcal{O}$ \\
\hline $\mathscr{G} \mathbb{E}$ & $\Sigma$ \\
\hline$\delta_{\alpha}^{o}$ & $\delta_{V}^{o}$ \\
\hline
\end{tabular}

The set of the subalgebras $\downarrow \mathscr{A}$ comes from the set $\mathscr{V}(\mathscr{H})$ of commutative subalgebras of the algebra $\mathscr{B}(\mathscr{H})$ of bounded operators on a Hilbert $\mathscr{H}$.

The contexts $\alpha$ come from the subalgebras $V \in \mathscr{V}(\mathscr{H})$.

The characteristic functions $\chi_{A}, A \in \mathscr{A}$ that represent the propositions can be found in Hilbert's orthogonal projectors.

The distributions $P$ are put into relationship with the $\mathscr{H}$ unit vectors.

$\mathbb{E}$ is the transposition of the external presheaf $\mathcal{O}$.

Let $J$ be a projector of $\mathscr{H}$ and $V$ ua commutative subalgebra of operators, the original definition of daseinification $\delta_{V}^{o}(J)$ is the largest projector of $V$ which is smaller than $J$. In the model presented here, the projector $J$ becomes a characteristic function $\chi_{B}$ with $B \in \mathscr{A}$. By using the same definition, we find $\delta_{\alpha}^{o}\left(\chi_{B}\right)=\max \left\{\chi_{A} \mid A \in \alpha, \chi_{A} \leqslant \chi_{B}\right\}=\chi_{\sum_{\Delta \in \pi \alpha \mid B \supseteq \Delta} \Delta .}$. It can be null.

The presheaf of spectral states $\Sigma$, which instantiates the observables, can be assimilated to the functor generating the Gel'fand spectra $\mathscr{G} \mathbb{E}$, something which isn't a problem because the $\mathbb{E} \alpha$ are commutative. 


\section{Conclusion}

This exercise throws new light on the notion of conditional mathematical expectation. The introduction of these contexts makes it possible to put classic and intuitionist uncertainties together via measurement results. We were able to explain the intuitionistic validation crible by introducing the orthogonal concepts of capacity and reservation. The notion of topology underlies this work. It would be interesting to research Grothendieck's topologies for the topos $\widehat{\downarrow \mathscr{A}}$. 


\section{Selected bibliography}

[1] A. Döring and C. Isham, New Structures for Physics Part V 13, Springer 2011.

[1.1] J. Gutt, Topoi for Quantum Mechanics, Warsaw University of Technology, 2017

[2] Cecilia Flori, A First Course in Topos Quantum Theory, Springer 2013.

[2.1] Cecilia Flori, A Second Course in Topos Quantum Theory, Springer 2018.

[3] M. Métivier, Notions Fondamentales de la Théorie des Probabilités, Dunod 1968.

[3.1] R. Steyer \& W. Nagel, Probability and Conditional Expectation, Wiley 2017.

[4] Yaglom \& Yaglom, Probabilité et Information, Dunod 1969. 


\section{List of symbols}

\begin{tabular}{|c|c|}
\hline Symbol & Definition \\
\hline$\Theta$ & state space \\
\hline $\boldsymbol{\theta}$ & element of $\Theta^{M}$ \\
\hline$\tilde{\boldsymbol{\theta}}$ & union of $\boldsymbol{\theta}$ coordinates \\
\hline $\mathscr{A}$ & $\sigma$-algebra on $\Theta$ \\
\hline$P$ & probability on $\mathscr{A}$ \\
\hline$\downarrow \mathscr{A}$ & lattice of finite subalgebras of $\mathscr{A}$ \\
\hline$\alpha, \beta$ & elements of $\downarrow \mathscr{A}$, contexts \\
\hline$\Delta, \Gamma$ & (resp.) atoms de $\alpha, \beta$ \\
\hline$\pi \alpha$ & partition of $\Theta$ by atoms of $\alpha$ \\
\hline $\mathbb{E}(X \mid \alpha)$ & conditional expectation of $X$ given the subalgebra $\alpha$ \\
\hline$\widehat{\downarrow \mathscr{A}}=\mathbf{S e t}^{\downarrow \mathscr{A}^{o p}}$ & topos of presheafs on $\downarrow \mathscr{A}$ \\
\hline $\mathbb{E}$ & presheaf of conditional expectations \\
\hline $\mathbb{E} \alpha$ & vector-space of $\alpha$-mesurables observables \\
\hline$\chi_{\Delta}$ & characteristic function of $\Delta$, basis vector of $\mathbb{E} \alpha$ \\
\hline$\chi^{\Delta}$ & dual form \\
\hline$s_{D}$ & subobject of $\mathbb{E}$ generated by the set of obervables $D$ \\
\hline$\Omega$ & subobject classifier of the topos $\widehat{\downarrow \mathscr{A}}$ \\
\hline$\chi_{s}$ & characteristic arrow of the subobject $s$ \\
\hline$G_{\boldsymbol{\theta}}^{\alpha}$ & measurement result of $\boldsymbol{\theta}$ in the context $\alpha$ \\
\hline $\mathscr{V}(G)$ & filter of propositions validated by $G$ \\
\hline$v_{I}^{\alpha}(B)$ & intuitionistic validity of $B$ in context $\alpha$ given the result $G_{\boldsymbol{\theta}}^{\alpha}$ \\
\hline$v_{C}^{\alpha}(B)$ & classic validity of $B$ in the context $\alpha$ given the result $G_{\boldsymbol{\theta}}^{\alpha}$ \\
\hline$\stackrel{\circ}{\alpha}, \partial \alpha, \stackrel{e}{\alpha}$ & (resp.) interior, border and exterior operators \\
\hline$\kappa$ & carrying capacity \\
\hline$G_{i}, G_{b}, G_{e}$ & (resp.) interior, border and exterior parts of the measurement \\
\hline
\end{tabular}




\begin{tabular}{|l|l|}
\hline Symbol & Definition \\
\hline$R=G_{e}+\bar{G}_{e}$ & reservation \\
\hline$M$ & number of subsystems \\
\hline$p_{s}$ & Wiener's transition probability \\
\hline$H(\alpha)$ & entropy of the context $\alpha$ \\
\hline$L_{i}, i \in D$ & numbers of linear divisions in dimension $D$ \\
\hline
\end{tabular}

